\title{
LA FORMACIÓN DE BURBUJAS ESPECULATIVAS Y LA CRISIS FINANCIERA: TEORÍA Y POLÍTICAS DE PREVENCIÓN*
}

Magnolia Miriam Sosa Castro ${ }^{1}$

\section{Resumen}

Durante las últimas tres décadas, las crisis financieras han sido fenómenos cada vez más recurrentes, profundos y de mayor magnitud. Los resultados sociales de las mismas han sido devastadores en términos de desarrollo y desigualdad económicos. En este contexto, el presente trabajo tiene por objetivo conceptualizar sobre la formación de las burbujas especulativas y su relación con crisis financieras, exponiendo las principales teorías e ideas entorno a dichos fenómenos. La hipótesis que se desprende de este análisis es que la formación de burbujas especulativas y crisis responden a la naturaleza del sistema de mercado. Dada la trascendencia que entrañan, tanto las crisis como las burbujas especulativas, se proponen algunas políticas de prevención y regulación con miras a una reforma del sistema financiero que fomente el crecimiento económico, disminuyendo la incertidumbre tanto a nivel nacional como a nivel internacional.

Palabras clave: burbujas especulativas, crisis financieras, políticas de prevención, teoría económica.

\section{Abstract}

During the last three decades, financial crises have been more frequent, deep and larger in magnitude. Their results have been devastating in social terms, impacting economic development and inequality. In this regard, the document aims to associate speculative bubbles formation

1 Profesora-Investigadora, del Departamento de Economía, de la Universidad Autónoma Metropolitana, Unidad Iztapalapa.

* Fecha de recepción: 21/06/2018. Fecha de aceptación: 27/11/2018. 
to financial crisis. To achieve this purpose, the main theories and ideas around those topics are exposed. The hypothesis stands that speculative bubbles and crises formation respond to market system dynamics. Due to the importance of financial crisis and speculative bubbles, prevention and regulation policies are proposed in order to promote financial system reforms aimed to enhance economic growth and diminish uncertainty in financial markets.

Key words: speculative bubbles, financial crisis, prevention policies, economic theory.

\section{Formación de burbujas especulativas: teorías y modelos}

Dentro del contexto financiero global contemporáneo, se presenta un fenómeno de manera recurrente, en el cual se muestran períodos de continuas alzas en precios con una marcada tendencia a sobrevalorarlos, esto es, dando lugar a la compra de un instrumento pagando un sobreprecio en relación con su valor real. La explicación de dicho fenómeno subyace en que el propietario tiene la expectativa de permanencia de la tendencia alcista, de tal manera que, la venta futura de su activo sea a un mayor precio, reportando una ganancia únicamente por su compra y venta, no así por los rendimientos asociados al valor fundamental de la inversión (valor actual de los flujos de caja medidos a partir de dividendos, intereses, tasas de descuento, etc.). A dicho fenómeno se le conoce como burbuja financiera ya que se caracteriza por una inflación en los precios, cuya explicación se encuentra en el interés por especular con un activo. En los estudios asociados con crisis, se define a las burbujas financieras como aquellos fenómenos en los que preceden a las crisis, una vez que la sobrevaloración llega a un punto máximo, los precios se desploman ocasionando que estalle la burbuja.

La sobrevaloración del precio de los activos financieros es perpetuada a través de su retroalimentación ya que se arguye que, si bien existen caídas abruptas en los precios de los activos, el mercado financiero está caracterizado por mantenerse en equilibrio, por lo cual, 
dichas disrupciones en el crecimiento de los precios no son más que desviaciones de la tendencia de largo plazo. La idea anterior es aquella que se encuentra detrás de la hipótesis de los mercados eficientes, desarrollada por Eugene Fama en 1970, en la cual se señala que, los precios de los activos reflejan toda la información disponible, las variaciones sucesivas en los precios son independientes y las variaciones esperadas corresponden a un juego justo. De manera estricta, las variaciones en los precios siguen un proceso de submartingala, en el cual la variación esperada puede llegar a ser positiva (Aragonés y Mascareñas, 1994; Bodie, Kane y Marcus, 2011).

Las burbujas financieras se pueden explicar, en primer lugar, a partir de un enfoque "macro" que es consistente con lo antes expuesto, ya que involucra al equilibrio general y expectativas racionales. De acuerdo con este enfoque, las burbujas ayudan a explicar y solventar un problema de depósito de valor en el mercado, en el corto plazo (Uribe y Ulloa, 2014). Samuelson (1958) señala que las burbujas pueden existir en modelos de generaciones traslapadas y que estas ayudan a resolver problemas de ineficiencia dinámica; por ejemplo, el dinero fiduciario es visto como una burbuja cuya función es la transferencia de recursos intrageneracionales. Así, el fenómeno se analiza como un aspecto necesario, sin el cual no sería posible dicha transferencia (Uribe y Ulloa, 2014). En línea con lo previamente señalado, Brunnermeier y Julliard (2008) proponen que la formación de burbujas especulativas se puede deber a ausencia de límites al arbitraje y desinformación de algunos agentes en el mercado, que desconocen que un activo se aleja de su valor fundamental.

En segundo lugar, de forma contrapuesta a la perspectiva "macro", existe un enfoque "micro" el cual apunta al no cumplimiento del supuesto de racionalidad, señalando dicha condición como el origen de la sobrevaloración de activos y de la formación de burbujas financieras (Case, Quigley y Shiller, 2005; Swedberg, 2010). A este respecto, algunos autores como Garcia (2015), Lei, Noussair y Plott (2001) y Hirshleifer (2015) explican la valoración de activos y la formación de burbujas especulativas a través de aspectos de índole psicológica, re- 
lacionados con el exceso de confianza, euforia y entusiasmo por las apuestas a realizar en el mercado; estas ideas base son los principios de las finanzas conductuales o behavioral finance (Kahneman y Tversky, 2013; Baddeley, 2018).

Otra propuesta de análisis explicativo, dentro del enfoque conductual, es la sugerida por Allen y Gale (2000) quienes atribuyen la formación de burbujas especulativas al problema de la agencia, en el cual los agentes tienen incentivos para decidirse por proyectos sumamente riesgosos, ya que no dimensionan ni enfrentan las consecuencias sociales de sus actos. Así, los agentes se deciden por proyectos riesgosos y el peor resultado esperado es la declaración en insolvencia (Uribe y Ulloa, 2014). Como confirmación de los efectos nocivos de las burbujas especulativas, los estudios de Geanakoplos, Axtell, Farmer et al. (2012) y Farmer (2013) aportan evidencia sobre el impacto negativo de las burbujas especulativas en la asignación de los factores en la economía; tema íntimamente relacionado con la socialización de las pérdidas financieras, ampliación de brecha en ingresos y pobreza.

De Long, Shleifer, Summers y Waldmann (1990) y Hong y Stein (1999) proponen que la sobrevaloración de activos puede deberse a que los inversionistas consideran como referencia la información pasada para la toma de decisiones. Al observar una tendencia positiva, se asume que continuará el alza en los precios y un grupo de inversores (feedback traders) seguirá comprando. El incremento en la demanda provoca que los precios aumenten y se reafirma la creencia de la tendencia positiva (Madrid y Hierro, 2015).

Daniel, Hirshleifer y Subrahmanyam (1998) apuntan que el sesgo entre el valor real y el de mercado se debe a una confirmación de la opinión positiva de un inversionista sobre un cierto activo, a través de información pública o de una señal del exterior, lo que le incentiva a seguir comprando a un precio, cada vez, mayor. Miller (1977) y Scheinkman y Xiong (2003) atribuyen el proceso de sobrevaloración de activos a opiniones disimiles entre los inversionistas optimistas y pesimistas. Si estos 
últimos mandan señales de no estar vendiendo al descubierto ${ }^{2}$, quiere decir que no prevén que haya cambios a la baja (Madrid y Hierro, 2015).

De la Torre e Ize (2011) describen cuatro diferentes paradigmas sobre el comportamiento de los agentes en relación a las burbujas especulativas a) el paradigma colectivo cognitivo, en el que nadie sabe qué sucede, b) el paradigma de información asimétrica, algunos entienden mejor que otros, c) el paradigma de reforzamiento costoso, en el que todos saben qué sucede pero nadie hace nada, ya que se da por sentado que existen de forma natural algunas burbujas y crisis dentro del sistema y d) el paradigma de acción colectiva, todos entienden lo que ocurre, pero no se hace nada al respecto ya que los intereses privados no son consistentes con aquellos de la sociedad.

Shiller (2000) menciona que una de las causas de las burbujas especulativas es el denominado efecto rebaño que se refiere al fenómeno en el que los individuos actúan de común acuerdo con lo que el resto lleva a cabo, a pesar de que vaya en contra de sus propias creencias. Una de las explicaciones a este fenómeno es que existen individuos que no tienen los recursos para allegarse de información en la toma de decisiones, así que, sigue la conducta del mercado, promovida generalmente por grandes inversionistas.

A este respecto, desde la década de 1970 con el abandono del sistema de Bretton Woods y la caída del patrón oro se registraron cambios importantes en el orden económico global, los cuales han trastocado de manera significativa la estabilidad de las variables financieras, induciendo burbujas financieras y a su vez modificando la naturaleza de las crisis e intensificando su impacto en la actividad económica internacional. De acuerdo con Ahmed (2009), Ferguson (2008) y Marichal (2010) las crisis sistémicas tienen una estrecha relación con las burbujas especulativas al ser precedidas por estas, por lo cual, la detección de burbujas especulativas puede ser un factor importante para la prevención de crisis.

2 La venta al descubierto es aquella que se lleva a cabo sin previa compra de los títulos. El objetivo es especular a partir de una disminución del precio en el corto plazo. 
Tal ha sido la importancia de las burbujas especulativas en las crisis financieras que Reinhart y Rogoff (2011) realizan una clasificación de cuatro tipos de crisis financieras: las de deuda pública, las de tipos de cambio, las de hiperinflación y las de crédito. Reconociendo en aquellas de hiperinflación las que se encuentran relacionadas con el proceso de sobrevaloración de activos y de formación de burbujas financieras.

\section{Determinantes de las crisis financieras: el papel de las burbujas especulativas en el contexto financiero contemporáneo}

Las crisis económicas son fases del ciclo en las que se da un decrecimiento de la actividad económica, siendo el periodo en el cual variables clave (producción, empleo, ingreso, etc.) presentan signos negativos. Si bien las crisis son inherentes a la actividad económica, no es posible saber cuándo ocurrirán. En esta dirección, se han desarrollado numerosos trabajos con el objetivo de desentrañar la naturaleza de las crisis e incrementar el conocimiento entorno a dichos fenómenos, cuyos efectos en la actividad económica y bienestar social son catastróficos.

Los trabajos sobre crisis económicas y los factores determinantes de las mismas son abundantes y muy diversos. Respecto a los elementos que las causan, se pueden dividir en reales y nominales. Dentro de la primera categoría se encuentran: la actividad comercial e industrial (Juglar, 1863); el nivel de inventarios (Kitchin, 1923); las cosechas agrícolas y su relación con el clima (Jevons, 1975); el subconsumo, que se da cuando la oferta incrementa, en mayor proporción que la demanda (Hobson, 1922; Fullarton, 1845; Sismondi, 1827; y Rodbertus, 1946); la innovación y aquello relacionado con el empresario y su incidencia en el cambio tecnológico (Schumpeter,1935).

Por otro lado, los movimientos cíclicos también han sido atribuidos a variables de tipo nominal: crédito y dinero (Hawtrey, 1913; Mises, 1912 y; Hayek, 1929 y 1931³), políticas económicas que contraen la 
base monetaria real (Friedman y Schwartz, 1963), factores aleatorios de tipo exógeno (Slutzky, 1937; Frisch et al. 1933; Mandelbrot, 1997; Lucas, 1975 y 1977), la inversión, que depende de la eficiencia marginal del capital (Keynes, 1936), variaciones de la tasa de ganancia capitalista (Marx, 1867; Marx y Engels, 18944), por mencionar algunos de los más relevantes. En la misma línea de los factores nominales, el término de crisis financiera es utilizado para hacer referencia a una recesión económica, cuyo origen no se encuentra en la economía real, sino que proviene de fallos en el sistema financiero o monetario.

A partir de los cambios en el contexto financiero global, se han desarrollado teorías y modelos para tratar de explicar cuáles son los factores que generan las crisis financieras. Rodríguez (Rodríguez et al. 2008) señala que existen tres generaciones de modelos de crisis financieras. Los modelos de primera generación consideran que las crisis son inevitables, pero previsibles y que son generadas a partir de inconsistencia entre la política monetaria y política fiscal o la debilidad de variables macroeconómicas. Algunos de los modelos de primera generación han sido desarrollados por Salant y Henderson (1978), Krugman (1979), Flood y Garber (1984), Calvo (1987), Deming (1989), Krugman, et al. (1991) y Flood, Garber y Kramer (1996) y Dooley $(2000)^{5}$.

3 Estos últimos autores, pertenecientes a la denominada escuela austríaca, la cual hace hincapié en el papel de la inversión como mecanismo de propagación del ciclo, resaltando la participación del sector monetario a manera de un movimiento incentivador pro cíclico del crédito y la deuda interna, (Jürgensen y Dosende, 1993).

4 El pensamiento de Marx respecto a las crisis se encuentra desarrollado en su obra más importante El Capital que consta de tres volúmenes, el primero de ellos publicado en vida en 1867 y el segundo y el tercero publicados post mortem, por Engels en 1885 y 1894, respectivamente.

5 Algunas conclusiones importantes dentro de los modelos de primera generación son: a) la imposibilidad de financiación de déficit para las economías que fijan el tipo de cambio, Krugman (1979); y b) a medida que incrementen las reservas iniciales se retrasa el colapso del régimen, mientras que de modo contrario, si aumenta el crédito interno se acelera el proceso, siendo el crédito interno y las reservas iniciales dos variables sumamente importantes para no incurrir en crisis de balanza de pagos, Flood y Garber (1984). 
Los modelos de segunda generación fueron desarrollados en la década de 1990, periodo a partir del cual se presentaron crisis, pese a que las variables macroeconómicas no presentaban signos de debilidad y a que había aparente consistencia entre las políticas económicas. Los modelos de segunda generación, a diferencia de los anteriores, consideran a las crisis como eventos impredecibles e inevitables, cuya principal causante son las expectativas de crisis por parte de los inversionistas. Las expectativas de crisis se generan a partir de las señales o noticias que aparecen en el mercado, propiciando que haya un ambiente especulativo previo a la crisis. Cuando se generan expectativas negativas y un grupo significativo pierde la confianza y retira los capitales, sucede la crisis.

En los modelos de segunda generación, si bien las variables fundamentales no son las causantes de la crisis, si son decisivas en la magnitud de esta. Dentro de este tipo de modelos destacan aquellos desarrollados por Obstfeld (1996), Cole y Kehoe (1996, 2000), Sachs, Tornell y Velasco (1996), Calvo (2004), Chang y Velasco (1998), Drazen (2000), Palley (1994), Taylor y Rada (2003), Minsky (1982) y Jeanne y Masson (2000) 6 .

Los modelos de tercera generación surgieron a partir de la crisis asiática (1997-1998), ante la insuficiencia teórica de los modelos previos para explicarla. En aquella ocasión las variables macro no presentaban debilidades, no había inconsistencia en las políticas económicas, ni expectativas de crisis. Además de que ciertos países tuvieron crisis gemelas (monetarias y bancarias) y trillizas (además de las anteriores crisis de deuda $)^{7}$. Las expli-

6 Es importante señalar que la clasificación de modelos económicos es inclusiva y abarca tanto modelos ortodoxos, como heterodoxos. En el caso de los modelos de segunda generación, son incluidos todos aquellos que cumplan con la condición general de explicar las crisis a través de shocks endógenos, generados por los cambios de las expectativas, independientemente de la corriente teórica económica en la que se basen; asimismo en el caso de los modelos de primera y tercera generación.

7 El Fondo Monetario Internacional identifica cuatro tipos de crisis financieras: monetarias, bancarias, de deuda y de paradas repentinas. En el presente ensayo sólo se hace mención a las tres primeras, ya que se considera que las de paradas repentinas, caracterizadas por una interrupción en la actividad de los principales sectores económicos, son consecuencia de los primeros tipos de crisis 
caciones entorno a los modelos de tercera generación sobre las causas de las crisis financieras son más complejas y divergen en los factores causantes.

Los modelos presentados por Dooley (2000), Corsetti, Presenti y Proubissi (1999) señalan como causantes de las crisis a políticas gubernamentales incongruentes que generan sobreendeudamiento por situaciones de riesgo moral. Allen (2001) y Requeijo (2006) apuntan a que los problemas de agencia y la traslación de riesgos son los principales causantes de las crisis. Chang y Velasco (1998) y Radelet y Sachs (1998) consideran que el pesimismo autorrealizable de los prestamistas internacionales es decisivo en el desencadenamiento de episodios de inestabilidad financiera.

Algunos trabajos inspirados en la teoría de Minsky apuntan a que los flujos de capital, los tipos de cambio y las tasas de interés externas tienen un papel clave en la generación de depresiones económicas. En estos modelos se considera que los cambios en las variables antes mencionadas generan shocks endógenos y exógenos, como resultado de la apertura económica (Dimsky, 2016; Kregel, 1998; Akyüz, et al. 1998; Oreiro, 2005; Sordi y Vercelli, 2012).

Finalmente, una de las explicaciones que se ha dado al génesis de las crisis es la formación de burbujas especulativas y cracks o estallidos en las mismas, las cuales propician salidas masivas de capital que contraen el crédito, limitando la inversión y la actividad económica. Al respecto, Stockhamer (2016) explica la crisis financiera global de 2008 a partir de la falta de crecimiento en la tasa de ganancia dentro del sector real, lo que promovió el crecimiento económico sustentado en la formación de burbujas financieras, el endeudamiento en los hogares y las exportaciones. Igualmente, señala que la crisis fue ampliada a partir de la arquitectura de la política económica.

Wang y Wen (2012) resaltan el papel de las burbujas especulativas en las crisis financieras, ya que al estallar causan mayor incertidumbre $\mathrm{y}$ volatilidad en los precios que cambios en variables fundamentales. Carmassi, Gros y Micossi (2009) comentan que hay tres ingredientes esenciales para la formación de una burbuja especulativa y son: i) exce- 
so de liquidez en los mercados de capital, alimentada por desequilibrios globales y por el crecimiento masivo de activos financieros (Kindleberger y Aliber, 2005), ii) crecimiento del crédito para mantener una tendencia de apalancamiento (Eichengreen y Mitchener 2003), iii) innovación financiera, a través de nuevos mecanismos como la titularización de deuda y la creación de instrumentos, cada vez más difíciles de valuar.

En términos de prever y predecir las crisis, se ha desarrollado un extenso cuerpo de literatura empírica para identificar indicadores cuantitativos que permitan lograr dicho objetivo. Así, se ha logrado detectar que casi la mayoría de las crisis han sido precedidas por una combinación de dos fenómenos: incremento en el apalancamiento, seguido por una importante expansión del crédito y un crecimiento inusual en el precio de activos financieros (Borio y Lowe, 2002; Adalid y Detken, 2007; Alessi y Detken, 2018).

La expansión del crédito y el incremento en el apalancamiento pueden ser medidos a partir de la razón (deuda/capital). El riesgo asociado a dicho indicador es que los agentes se comprometen a cumplir con ciertas obligaciones, pero no cuentan con solvencia suficiente para hacerles frente. La manera de estimar dicho indicador en una perspectiva macroeconómica puede ser a partir de la relación crédito/PIB; sin embargo, no hay un límite o referente estandarizado para todas las economías, ya que dependiendo de sus características y fundamentos podrán soportar diferentes niveles (Carmassi, Gros y Micossi, 2009).

En relación con lo señalado por Stockhamer (2016), en la literatura financiera se hace referencia a una dinámica de financiarización la cual se explica a partir de la disminución de la tasa de ganancia en la inversión productiva, incitando a los agentes a realizar inversiones en el terreno financiero, aun cuando su fin último, en el caso de las empresas, sea otro muy distinto. Las inversiones financieras proveen de excesiva liquidez a los mercados, generando sobrevaloración de los activos, formación de burbujas financieras, el estallido de las mismas y volatilidad en las variables fundamentales. 
La disminución de la tasa de ganancia, que promueve la dinámica de financiarización, tiene su origen en el decremento del ingreso real, y se ve materializada a través de efectos negativos en la demanda (ver figura 1). De tal forma que, cuando se realizan inversiones productivas con la intención de incrementar la oferta, se genera una caída en los precios que impacta el margen de ganancia. Así, los inversionistas prefieren orientar sus inversiones hacia los mercados financieros, donde se obtendrán mayores beneficios (Ferrus y Rivas 2017) ${ }^{8}$. Para que la tasa de ganancia financiera no disminuya, se incentiva al crédito para generar el pago de intereses y estimular la demanda, que no puede ser sostenida por la contracción del ingreso real. El proceso es prolongado hasta que el crédito alcanza los límites del sobreendeudamiento y vulnera la estabilidad financiera, provocando el estallido de burbujas especulativas y crisis. El resultado del proceso antes detallado es la dominación del capital financiero sobre la actividad económica global (Serfati, 2000; Stockhammer, 2009; McNally, 2017).

\section{Figura 1. Crisis financieras y burbujas especulativas}

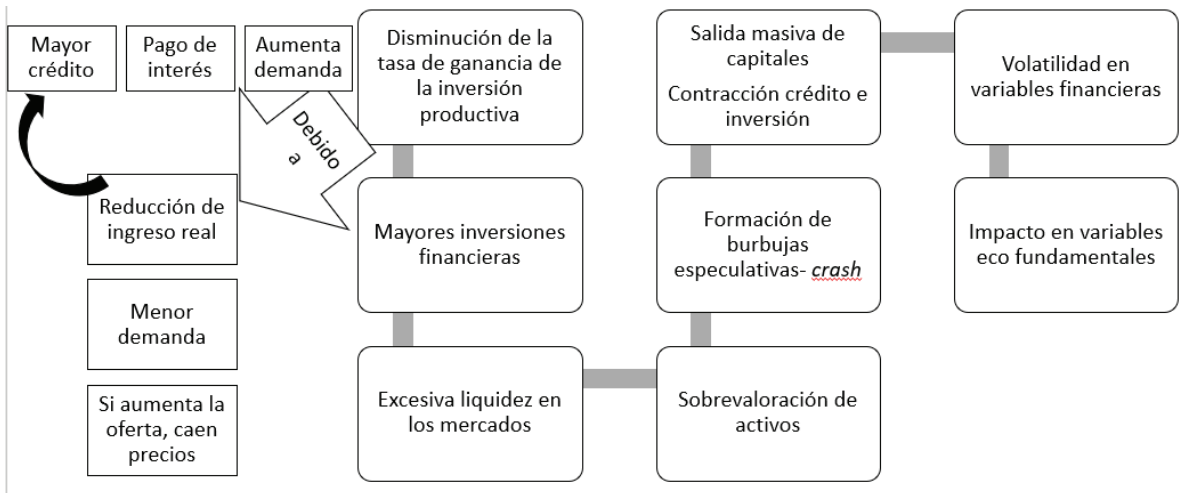

Fuente: elaboración propia con los conceptos desarrollados

8 La idea central de la financiarización es que existe una contradicción entre el capital financiero y el productivo (Duménil y Levy, 2004; Gowan, 1999; Plihon, 2001) 
Como señala Palley (2013) la era de la financiarización se caracteriza por la reorientación del ingreso hacia el capital, cambio en la composición de pagos al capital, incrementando la participación de los intereses y aumento en la participación del sector financiero en las ganancias a nivel global. El proceso de dominación financiera tiene importantes efectos en el bienestar social, introduciendo riesgo al sistema económico y acentuando la desigualdad ${ }^{9}$.

Ante las recientes crisis financieras (subprime, de la deuda soberana y financiera global) precedidas por la formación de burbujas financieras en el sector inmobiliario, especialmente, estadounidense y europeo, como respuesta a las mismas se ha puesto de manifiesto que existen debilidades y áreas de oportunidad importantes en materia de regulación y supervisión financieras. El principal reto es dotar al sistema financiero de fortaleza y certidumbre, promoviendo la confianza en el mismo, sin impedir su desarrollo, procurando el bienestar social y protegiendo el interés público, no dejando de lado la innovación dentro de un contexto financiero global íntimamente interconectado. Ante dicho reto, se han desarrollado diversas propuestas sobre políticas de prevención de formación de burbujas especulativas y crisis financieras.

\section{Políticas de prevención en el ámbito financiero ante la formación de burbujas especulativas y crisis}

Las propuestas provenientes de organismos internacionales y de los gobiernos de las principales 20 economías a nivel global han sido consensuadas en lo que se conoce como Junta de Estabilidad Financiera (FSB por sus siglas en inglés ${ }^{10}$ ) y se han visto materializadas en acuerdos internacionales, el más importante de ellos Basilea III, el cual con-

9 Una cantidad importante de recursos es obtenida a través del sistema financiero, por medio del sistema de pensiones e inversiones en el mercado de capitales y a través de altas tasas de interés por concepto de crédito hipotecario, automotriz y al consumo.

10 Financial Stability Boarder. 
templa los siguientes puntos: i) reforzar los requerimientos de capital y liquidez dentro del sistema bancario, ii) mejorar la administración de riesgo, iii) mejorar estándares contables, sobre las normas de información financiera internacional, iv) realizar reformas sobre aquellos derivados Over the Counter, v) adopción de principios para evitar incentivos perversos en la toma de decisiones riesgosas ${ }^{11}$, vi) crear una plataforma sobre las transacciones bilaterales de los principales bancos, vii) mejorar el modelo de bursatilización, viii) estrategias para reducir aquellas instituciones consideradas "muy grandes para caer" (Claessen y Kodres, 2014 y IMF, 2013)

Además de lo antes mencionado, Claessens y Kose (2013) y Giovanolli (2009) consideran que se debe: regular a las agencias calificadoras, estandarizar los modelos sobre la valoración de activos intangibles e instrumentos difíciles de valuar, someter a regulación a agencias crediticias, regular fondos de cobertura (hedge funds) y promover la creación de un sistema de alerta oportuna de crisis por parte de organismos financieros internacionales, por mencionar algunas de las acciones más importantes.

De acuerdo con Claessens y Kose (2013), la clave de la regulación y supervisión se encuentra en el diseño de las estrategias y en la velocidad de respuesta ante cambios e innovaciones en el sistema financiero. Se debe considerar que, en ocasiones cierto tipo de intervención gubernamental genera efectos de distorsión (Barth, Caprio y Levine, 2006), por ejemplo, los bancos al saberse pieza clave de la economía se sobre-apalancan; igualmente, las instituciones que se reconocen "muy grandes para caer" toman decisiones riesgosas, ya que ambos se consideran protegidos por el Estado. Lo anterior genera vulnerabilidad y fallos en la economía, contrario a lo que se persigue con la regulación macro y micro prudencial (Laeven, 2011).

En respuesta a lo anteriormente expuesto Shiller (2010) considera que se deben dejar de lado ese tipo de soluciones a las crisis, en las cua-

11 En este sentido, se han hecho propuestas como la regla Volcker que propone que los bancos no puedan realizar inversiones riesgosas ni participar en fondos de cobertura o empresas de capital de riesgos 
les se "premia" y "rescata" a los responsables. Lo que se propone es el rediseño del sistema financiero y no seguir poniendo "parches" ante su mal funcionamiento. La clave está en formar instituciones de calidad a partir de lo que se denomina democratización de las finanzas: ampliar la aplicación de principios financieros sólidos a un segmento cada vez mayor de la sociedad, utilizando toda la tecnología moderna, para lograr este objetivo. Una de las principales propuestas de Shiller frente a la crisis es la detección y racionalización de las burbujas especulativas, en lugar de intervenir de manera urgente con soluciones que emplean recursos públicos para resolver fallos ocasionados por agentes privados.

A este respecto, en términos de la economía conductual Pete (2014) considera que las políticas de regulación deben ir orientadas en dos sentidos: los consumidores y la competencia (evitando la formación de entidades "muy grandes para caer" cuya acción provoca distorsiones en el mercado). La primera dirección está relacionada con promover y demandar información conduciendo a una toma de decisiones más simplificada, con el objetivo de tener mejores y más convenientes opciones. Dentro de esta vertiente, se apela por incrementar la información en algunos sectores donde los consumidores enfrentan desventajas sistemáticas, tal es el caso de los fondos de pensiones. Igualmente, se hace hincapié en que debe modificarse la manera en que la información relacionada con los instrumentos financieros es comunicada, ya que al consumidor no le es posible identificar ni discernir entre aquella información relevante, para tomar la mejor decisión (Pete, 2014).

La propuesta de regulación prudencial, bajo los enfoques pragmáticos, reconocidos por la economía conductual (De la Torre e Ize, 2011), consiste en: a) regular aquellos intermediarios financieros apalancados, no solo aquellos de tipo sistémico b) fiscalizar intermediarios financieros que presenten inconsistencia entre los préstamos a realizar y las inversiones de las cuales recibirán beneficios, en el corto y largo plazo y c) promover asesoría dentro de los mercados financieros para que existan decisiones racionales y bien informadas. Las propuestas pueden resumirse en la figura 2 . 
Figura 2. Resumen de políticas de prevención

Propuesta principales organismos BM, FMI y G20
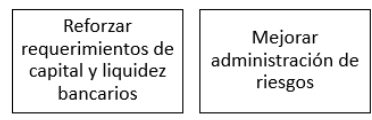

Mejorar en NIIF riesgos

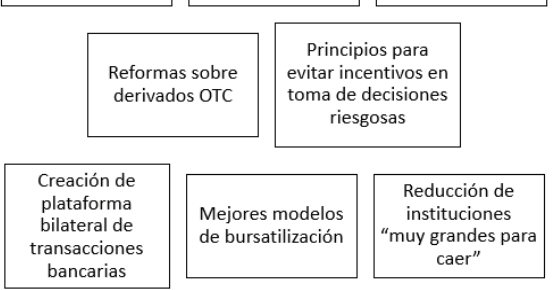

\section{Propuestas complementarias}
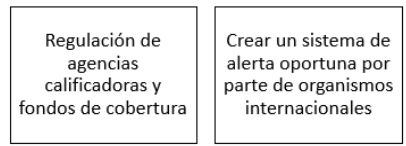

Democratización de las finanzas, mayor participación social

Incrementar la información, para una toma de decisiones más simplificada

Comunicación de la información financiera completa y objetiva para mayor control

Fuente: elaboración propia con base en la revisión previa.

\section{Conclusiones}

El objetivo del presente trabajo es conceptualizar sobre la formación de las burbujas especulativas y su relación con crisis financieras, exponiendo las principales teorías e ideas entorno a dichos fenómenos. Así, el trabajo en la primera sección define y teoriza sobre el concepto de burbuja especulativa y su relación con las crisis financieras, la segunda parte, desarrolla los modelos sobre formación de crisis (categorizadas en tres generaciones), la tercera parte expone las políticas de prevención de crisis financieras de los organismos internacionales y de algunos otros teóricos.

En términos de la hipótesis, la formación de burbujas especulativas $y$, sucesivas, crisis responde a la naturaleza del sistema de mercado. Se realiza una exposición de los aspectos vinculados a la dinámica financiera global, particularmente, el fenómeno de financiarización, en el cual los factores financieros dominan sobre aquellos de tipo productivo. Dicho análisis pone de relevancia la necesidad de replantear de forma crítica y objetiva al sistema económico, buscando formas alternativas que promuevan la redistribución del ingreso y la demanda, disminuyen- 
do la dependencia de la economía real hacia el sistema financiero. Para lograr dicho objetivo, es importante diseñar un marco regulatorio que sea implementado de la mano con una política fiscal, a modo de castigar todas aquellas actividades que vayan en detrimento de los intereses públicos. De modo contrario, las crisis seguirán ocurriendo, ya que hay un grupo que se favorece a través de éstas, al tomar decisiones riesgosas, para en lo sucesivo ser rescatado con recursos de la sociedad.

En esa dirección, el papel del Banco Central como garante del interés financiero debiera ser más activo, disminuyendo la distorsión que generan los grandes capitales en la instrumentación de política económica. Así, las autoridades económicas, deben emplear los instrumentos que tengan a su alcance para lograr objetivos particulares enfocados a la promoción del crecimiento y desarrollo económicos.

En relación a las políticas de regulación en países emergentes, como es el caso de México, además de los estándares a nivel global y la coordinación con organismos internacionales, es importante implementar medidas de control de formación de burbujas y prevención de crisis procurando el diseño de políticas que respondan a las necesidades propias de cada entorno en desarrollo y que vayan encaminadas a no profundizar las desigualdades sociales, para que así el mayor bienestar de la población se convierta en el pilar de las fortalezas institucionales. De manera simultánea a las medidas de política pública, es necesario seguir desarrollando aplicaciones enfocadas a la medición, supervisión y control de riesgos que permitan una detección temprana de los mismos y evitar que se materialicen como en las más recientes crisis financieras.

Finalmente, dentro de las líneas futuras de investigación, se podría realizar un estudio empírico analizado con la metodología de casos. 


\section{Bibliografía}

Adalid, Ramon, Detken, Carsten, Liquidity shocks and asset price boom/bust cycles, Working papers series, 2007.

Ahmed, Habib, "Financial crisis, risks and lessons for Islamic finance", ISRA International Journal of Islamic Finance, 2009, vol. 1, núm. 1, pp. 7-32.

Akyüz, Yilmaz, Chang, Ha-Joon, Kozul-Wright, Richard, "New perspectives on East Asian development", The Journal of Development Studies, 1998, vol. 34, núm. 6, pp. 4-36.

Alessi, Lucia, Detken,Carsten, "Identifying excessive credit growth and leverage", Journal of Financial Stability, vol. 35, 2018, pp. 215225 .

Allen, Franklin, "Financial structure and financial crisis", International Review of Finance, vol. 2, núm. 1 y 2, 2001, pp. 1-19.

Allen, F., \& Gale, D, "Financial contagion", Journal of political economy, vol. 108, núm. 1, 2000, pp. 1-33.

Aragonés, José R., Mascareñas, Juan, “La eficiencia y el equilibrio en los mercados de capital”, Análisis financiero, vol. 64, núm. 1, 1994, pp. 76-89.

Baddeley, Michelle, "Financial Instability and Speculative Bubbles: Behavioural Insights and Policy Implications", Alternative Approaches in Macroeconomics, Palgrave Macmillan, Cham, 2018, pp. 209-234.

Barth, James R., Caprio, Gerard, Levine, Ross, Rethinking bank regulation: Till angels govern, Cambridge University Press, 2006.

Bodie, Zvi, Kane, Alex, Marcus, Alan J., Investment and portfolio management, McGraw-Hill Irwin, 2011.

Borio, Claudio Ev, Lowe, Philip William, Asset prices, financial and monetary stability: exploring the nexus, Working Papers, 2002. 
Brunnermeier, Markus K., Julliard, Christian, "Money illusion and housing frenzies", The Review of Financial Studies, 2008, vol. 21, núm. 1, pp. 135-180.

Calvo, Guillermo A., "Balance of payments crises in a cash-in-advance economy", Journal of Money, Credit and Banking, 1987, vol. 19, núm. 1, pp. 19-32.

Calvo, Guillermo A., "Contagion in emerging markets: when Wall Street is a carrier", en Latin American Economic Crises, Palgrave Macmillan, London, 2004, pp. 81-91.

Carmassi, Jacopo, Gros, Daniel, Micossi, Stefano, "The global financial crisis: Causes and cures", JCMS: Journal of Common Market Studies, 2009, vol. 47, núm. 5, pp. 977-996.

Case, Karl E., Quigley, John M., Shiller, Robert J, “Comparing wealth effects: the stock market versus the housing market", Advances in macroeconomics, 2005, vol. 5, núm. 1.

Chang, Roberto, Velasco, Andres, The Asian liquidity crisis, National bureau of economic research, 1998.

Claessens, Stijn, Kodres, Ms Laura E., The regulatory responses to the global financial crisis: Some uncomfortable questions, International Monetary Fund, 2014.

Claessens, Stijn, Kose, Mr M. Ayhan, Financial crises explanations, types, and implications, International Monetary Fund, 2013.

Cole, Harold L., Kehoe, Timothy J., "A self-fulfilling model of Mexico's 1994-1995 debt crisis", Journal of international Economics, vol. 41, núm. 3 y 4, 1996, pp. 309-330.

, "Self-fulfilling debt crises", The Review of Economic Studies, vol. 67, núm. 1, 2000, pp. 91-116.

Corsetti, Giancarlo, Pesenti, Paolo, Roubini, Nouriel, "The Asian crisis: an overview of the empirical evidence and policy debate", The Asian financial crisis: Causes, contagion and consequences, 1999, pp. 127-66. 
Daniel, Kent, Hirshleifer, David, Subrahmanyam, Avanidhar, "Investor psychology and security market under-and overreactions", The Journal of Finance, vol. 53, núm. 6, 1998, pp. 1839-1885.

De La Torre, Augusto, Ize, Alain, Containing systemic risk: paradigm-based perspectives on regulatory reform, The World Bank, 2011.

De Long, J. Bradford, et al., "Noise trader risk in financial markets", Journal of political Economy, vol. 98, núm. 4, 1990, pp. 703-738.

Deming, W. Edwards, Calidad, productividad y competitividad: la salida de la crisis, Ediciones Díaz de Santos, 1989.

Dymski, Gary, The Bank Merger Wave: The Economic Causes and Social Consequences of Financial Consolidation: The Economic Causes and Social Consequences of Financial Consolidation, Routledge, 2016.

Dooley, Michael P, "A model of crises in emerging markets", The economic journal, vol. 110, núm. 460, 2000, pp. 256-272.

Drazen, Allan, "Political contagion in currency crises", en Currency crises, University of Chicago Press, 2000, pp. 47-67.

Eichengreen, Barry, Mitchener, Kris James, The Great Depression as a credit boom gone wrong, 2003.

Farmer, Roger Ea, Nourry, Carine, Venditti, Alain, The inefficient markets hypothesis: why financial markets do not work well in the real world, National Bureau of Economic Research, 2012.

Ferguson, Niall, "El auge del dinero", Letras libres, 2008.

Ferruz y Rivas, Las burbujas financieras. Inversion y desinversion, Bubok, 2017.

Flood, Robert P., Garber, Peter M., "Collapsing exchange-rate regimes: some linear examples", Journal of international Economics, vol. 17, núm. 1 y 2, 1984, pp. 1-13. 
Flood, Robert P., Garber, Peter M., Kramer, Charles, "Collapsing exchange rate regimes: Another linear example", Journal of International Economics, vol. 41, núm. 3 y 4, 1996, pp. 223-234.

Friedman, Milton; Schwartz, Anna, A monetary history of the United States, The United States, 1963.

Frisch, Ragnar, et al., "Propagation problems and impulse problems in dynamic economics", Cambridge University Press, 1933, pp. 333346.

Fullarton, John, On the Regulation of Currencies... with corrections and additions, John Murray, 1845.

García Blanco, José María, "Burbujas especulativas y crisis financieras. Una aproximación neofuncionalista", Revista Española de Investigaciones Sociológicas (REIS), vol. 150, núm. 1, 2015, pp. 71-87.

Geanakoplos, John, et al., "Getting at systemic risk via an agent-based model of the housing market", American Economic Review, vol. 102, núm. 3, 2012, pp. 53-58.

Giovanoli, Mario, The reform of the international financial architecture after the global crisis, NYUJ Int'l L. \& Pol., vol. 42, 2009, p. 81.

Guzmán Vásquez, Alexander, Trujillo Dávila, María Andrea, "Burbujas en los precios de los activos financieros", Pensamiento \& Gestión, núm. 24, 2008, pp. 63-87.

Hawtrey, Ralph G, Good and Bad Trade: An Inquiry into the Causes of Trade Fluctuations, Reprint, Augustus M. Kelley, New York, 1913.

Hayek, Friedrich August, Geldtheorie und Konjunkturtheorie, Hölder-Pichler-Tempsky ag, 1929.

Hayek, Prices and Production, Routledge \&amp; Sons, Londres, 1931 [trad. esp.: Precios y producción, Ediciones Aosta/Unión Editorial, 1996].

Hirshleifer, David, "Behavioral finance", Annual Review of Financial Economics, 2015, vol. 7, pp. 133-159. 
Hobson, John Atkinson, Incentives in the new industrial order, L. Parsons, London, 1922.

Hong, Harrison, Stein, Jeremy C., "A unified theory of underreaction, momentum trading, and overreaction in asset markets", The Journal of finance, vol. 54, núm. 6, 1999, pp. 2143-2184.

International Monetary Fund, Key Aspects of Macroprudential Policy, Board paper (Washington: International Monetary Fund), 2013.

Jeanne, Olivier, Masson, Paul, "Currency crises, sunspots and Markov-switching regimes", Journal of international economics, vol. 50, núm. 2, 2000, pp. 327-350.

Jevons, F. R., "But some kinds of knowledge are more equal than others", Journal Studies in Science education, vol. 2, 1975, pp. 165.173 .

Juglar, Clément, Crises comerciales, Vve Berger-Levrault, 1863.

Jürgenson, K, Rosende R. Francisco, Hayek y el ciclo económico: una revisión a la luz de la macroeconomía moderna, Pontificia Universidad Católica de Chile, Instituto de Economía, Oficina de Publicaciones, 1993.

Kahneman, Daniel, Tversky, Amos, "Choices, values, and frames", en Handbook of the Fundamentals of Financial Decision Making, Part I, 2013, pp. 269-278.

Keynes, John Maynard, "The general theory of money, interest and employment", Reprinted in The Collected Writings of John Maynard Keynes, vol. 7, 1936.

Kindleberger, Charles P.; Aliber, Robert Z., Manias, panics, and crashes, Hoboken. 2005.

Kregel, Jan A., et al., Yes, "It" did happen again-a Minsky crisis happened in Asia, EconWPA, 1998.

Kitchin, J."Cycles and trends in economic factors", Review of Economics and Statistics, Vol. 5, No.1, 1923, pp. 10-16. 
Krugman, Paul, "A model of balance-of-payments crises", Journal of money, credit and banking, vol. 11, núm. 3, 1979, pp. 311-325.

Krugman, Paul, et al., "International aspects of financial crises", en The Risk of Economic Crisis, University of Chicago Press, 1991, pp. 85134.

Laeven, Luc, "Banking crises: A review", Annu. Rev. Financ. Econ., vol. 3, núm. 1, 2011, pp. 17-40.

Lei, Vivian, Noussair, Charles N., Plott, Charles R., "Nonspeculative bubbles in experimental asset markets: Lack of common knowledge of rationality vs. actual irrationalit", Econometrica, vol. 69, núm. 4, 2001, pp. 831-859.

Lucas Jr, Robert E., "An equilibrium model of the business cycle", Journal of political economy, vol. 83, núm. 6, 1975, pp. 1113-1144.

Lucas, Robert E., "Understanding business cycles", Carnegie-Rochester conference series on public policy, vol. 5, North-Holland, 1977, pp. 7-29.

Madrid, Alberto, Hierro, Luis A., "Burbujas especulativas: El estado de una cuestión poco estudiada", Cuadernos de Economía, vol. 38, núm. 108, 2015, pp. 123-138.

Malkiel, Burton G., Fama, Eugene F., "Efficient capital markets: A review of theory and empirical work", The journal of Finance, vol. 25, núm. 2, 1970, pp. 383-417.

Mandelbrot, Benoit B., "The variation of certain speculative prices", Fractals and scaling in finance, Springer, New York, 1997, pp. 371-418.

Marichal, Carlos, "Las grandes crisis financieras", Introducción y Capítulo, vol. 6. 2010.

Marx, Karl, El Capital, vol. I., 1867.

Marx, Karl, Engels, Friedrich, Capital: a critique of political economy. Book 3, The process of capitalist production as a whole, Lawrence \& Wishart, 1894. 
McNally, David, "From financial crisis to world slump: Accumulation, financialization, and the global slowdown", Karl Marx, Routledge, 2017, pp. 133-154.

Miller, Edward M., "Risk, uncertainty, and divergence of opinion", The Journal of finance, vol. 32, núm. 4, 1977, pp. 1151-1168.

Minsky, Hyman P., Inflation, recession and economic policy, Wheatsheaf Books, Brighton, Sussex, 1982.

Mises, Ludwig von, The theory of money and credit, Liberty Fund, Indianapolis, 1912.

Obstfeld, Maurice, "Models of currency crises with self-fulfilling features", European economic review, vol. 40, núm. 3-5, 1996, pp. 1037-1047.

Oreiro, José Luís, "Capital mobility, real exchange rate appreciation, and asset price bubbles in emerging economies: a Post Keynesian macroeconomic model for a small open economy", Journal of Post Keynesian Economics, vol. 28, núm. 2, 2005, pp. 317-344.

Palley, Thomas I, "Debt, aggregate demand, and the business cycle: an analysis in the spirit of Kaldor and Minsky", Journal of Post Keynesian Economics, vol. 16, ním. 3, 1994, pp. 371-390.

Palley, Thomas I, "Financialization: What it is and Why it Matters", en Financialization, Palgrave Macmillan, London, 2013, pp. 17-40.

Pete, Lunn, Regulatory policy and behavioural economics, OECD Publishing, 2014.

Radelet, Steven, Sachs, Jeffrey, The onset of the East Asian financial crisis, National bureau of economic research, 1998.

Reinhart, Carmen M., Rogoff, Kenneth S., "From financial crash to debt crisis", American Economic Review, vol. 101, núm. 5, 2011, pp. 1676-1706.

Requeijo, Jaime, Economía española, Delta Publicaciones, 2006. 
Rodbertus, Karl, Die Forderungen der arbeitenden Klassen, V. Klostermann, 1946.

Rodil Marzábal, Oscar, Menezes Ferreira, Vicente, "La crisis financiera global en perspectiva: génesis y factores determinantes", Revista de Economía Mundial, vol. 31, 2012.

Rodríguez Castellanos, Arturo, Urionabarrenetxea Zabalandikoetxea, Sara, San Martín Albizuri, Nerea, "Crisis financieras y globalización: un análisis de sus factores determinantes", Problemas del desarrollo, vol. 39, núm. 153, 2008, pp. 159-183.

Sachs, Jeffrey, Tornell, Aaron, Velasco, Andres, Financial crises in emerging markets: the lessons from 1995, National bureau of economic research, 1996.

Salant, Stephen W., Henderson, Dale W., "Market anticipations of government policies and the price of gold", Journal of political economy, vol. 86, núm. 4, 1978, pp. 627-648.

Samuelson, Paul A., "An exact consumption-loan model of interest with or without the social contrivance of money", Journal of political economy, vol. 66, núm. 6, 1958, pp. 467-482.

Scheinkman, Jose A., Xiong, Wei, "Overconfidence and speculative bubbles", Journal of political Economy, vol. 111, núm. 6, 2003, pp. 1183-1220.

Schumpeter, Joseph, "Análisis del cambio económico", The Review Economics Statistics, vol. XVII, núm. 4, 1935.

Serfati, Claude, "Le bras armé de la mondalisation", Temps modernes, núm. 607, 2000, pp. 41-64.

Shiller, Robert J., “El estallido de la burbuja”, Gestión, vol. 2009, 2000, p. 140 .

Shiller, Robert J, "How should the financial crisis change how we teach economics?", The Journal of Economic Education, vol. 41, núm. 4, 2010, pp. 403-409. 
Sismondi, Jean Charles Léonard, Nouveaux principes d'économie politique, ou de la richesse dans ses rapports avec la population, Delaunay, 1827.

Slutzky, Eugen, "The summation of random causes as the source of cyclic processes", Econometrica: Journal of the Econometric Society, 1937, pp. 105-146.

Sordi, Serena, Vercelli, Alessandro, "Heterogeneous expectations and strong uncertainty in a Minskyian model of financial fluctuations", Journal of Economic Behavior \& Organization, vol. 83, núm. 3, 2012, pp. 544-557.

Stockhammer, Engelbert, "The finance-dominated accumulation regime, income distribution and the present crisis", European Papers, vol. 19, 2009, pp. 58-81.

Stockhammer, Engelbert, Neoliberal growth models, monetary union and the Euro crisis. A post-Keynesian perspective", New Political Economy, vol. 21, núm. 4, 2016, pp. 365-379.

Swedberg, Richard, "The structure of confidence and the collapse of Lehman Brothers", en Markets on trial: The economic sociology of the US financial crisis: Part A. Emerald Group Publishing Limited, 2010, pp. 71-114.

Taylor, Lance, Rada, Codrina, "Debt-equity cycles in the 20th century: empirical evidence and a dynamic Keynesian model", Center for Economic Policy Analysis, New York, NY, New School University, 2003.

Uribe Gil, Jorge Mario, Ulloa Villegas, Inés María, "Burbujas financieras: dos alternativas de identificación aplicadas a Colombia", Sociedad y Economía, núm. 27, 2014, pp. 47-72.

Wang, Pengfei, Wen, Yi., "Speculative bubbles and financial crises", American Economic Journal: Macroeconomics, vol. 4, núm. 3, 2012, pp. 184-221. 
